\title{
Mesenteric chylous lymphangioma in the adult patient: a case report
}

\author{
Chris Mykytyshyn (Meds 2016), Dr Daniel Pepe (MD) \\ Faculty Reviewer: Dr Steven Latosinsky, MD, MSc, FRCSC (Department of Surgical Oncology)
}

\section{ABSTRACT}

Mesenteric lymphangiomas are a rare cause of intra-abdominal mass in the adult population. The incidence is estimated to be 1 in 100000 to 1 in 500000 patients. For this reason, the diagnosis of mesenteric lymphangioma is often overlooked on the differential diagnosis of abdominal mass. We describe the case of a 29-year-old Caucasian male who presented with abdominal distention, nausea, vomiting, jaundice, and constipation, who was ultimately diagnosed with mesenteric lymphangioma. Furthermore, we discuss the etiology and epidemiology, presentation and diagnosis, and treatment of mesenteric lymphangioma.

\section{INTRODUCTION}

Mesenteric lymphangiomas are fluid filled sacs which originate in the small bowel or large bowel mesentery and present as cystic intra-abdominal masses. These cysts may be pedunculated or sessile and hemorrhagic, serous, purulent, and/or chylous., ${ }^{1,2}$ Lymphangiomas are more prevalent in the pediatric population as they are often congenital malformations; presentation in the adult is exceedingly rare, with an estimated incidence of 1:100 000 to 1:500 000 patients. $^{3}$ There are several theories pertaining to the etiology of mesenteric lymphangioma, of which one involves the aberrant proliferation of lymphatic tissue following abdominal trauma. Due to its relatively low incidence in the adult population, the diagnosis of mesenteric lymphangioma can easily be overlooked; however, it should be kept on the differential diagnosis for any patient presenting with an intra-abdominal mass. With this consideration, we present a case of mesenteric chylous lymphangioma in an adult patient and review the relevant background information and clinical management.

\section{CASE REPORT}

A 29-year old Caucasian male presented initially to his local emergency department (ED) complaining of jaundice, nausea, vomiting, fever, and a distended abdomen. The patient stated that 4 days prior to his initial presentation he was shoveling snow from his driveway when he slipped on ice and fell onto his back. He reported no abdominal or gastrointestinal symptoms at the time of injury and proceeded to finish shoveling without complaint. Physical examination at that time revealed jaundice and a distended abdomen. He was subsequently admitted to his local hospital. The patient's jaundice resolved after several days and he was subsequently discharged.
Two weeks later, he returned to his local ED with complaints of significant abdominal pain and further abdominal distention, nausea, and vomiting. Physical examination at that time revealed abdominal distention and a large intra-abdominal mass on palpation. He was readmitted to his local hospital for acute pain management; a computed tomography (CT) scan and magnetic resonance imaging (MRI) of the abdomen were arranged.

Twenty-five days after his injury, the CT and MRI scans of the abdomen were performed. They revealed a large cystic mass within the midabdomen, measuring $30 \mathrm{~cm} \times 15 \mathrm{~cm}$. The radiologist reported that the mass appeared to abut several regions of small bowel including the third part of the duodenum. There appeared to be some calcifications within the mass in addition to possible septations. The differential diagnosis of the mass favoured giant lymphangioma but also included duplication cyst, gastrointestinal tumour, lymphocoele, and giant urachal cyst.

The patient was seen by a community general surgeon and a colonoscopy was performed, revealing no obvious pathology. The surgeon thus presented his case to the London Regional Cancer Program's Gastrointestinal Multidisciplinary Tumour Board Case Conference. The patient was to be referred to our general surgery service for management and we saw the patient 1 month after his initial injury.

In our initial interview, the patient stated that he had a 20-pound weight loss and was experiencing constipation, nausea, fatigue, and exertional dyspnea. He reported being otherwise

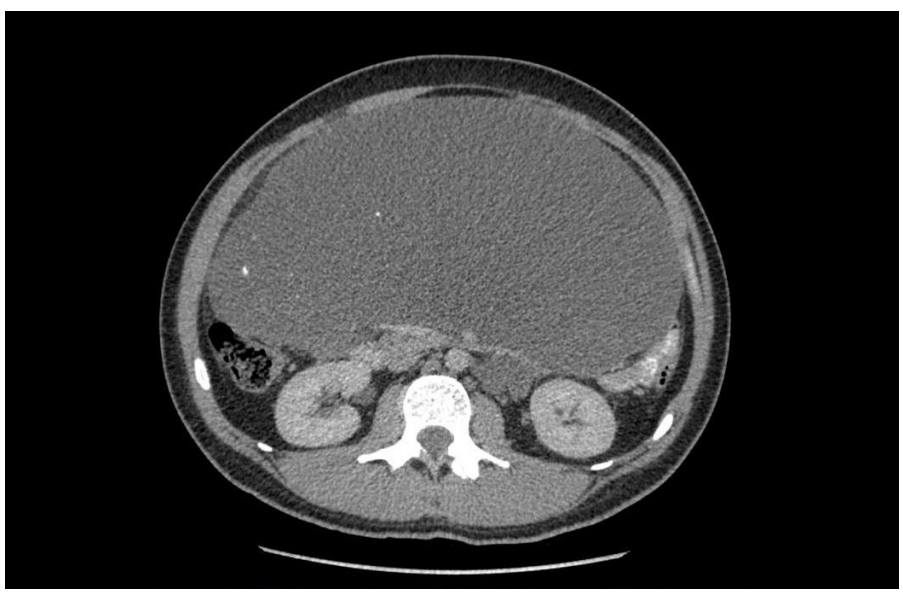

Figure 1. CT scan of the abdomen at the L2 level showing a large cystic mass with several calcifications seen as white hyperdensities. 
healthy with no significant past medical or surgical history. He was not taking any medications except Dilaudid $1 \mathrm{mg}$ every 4 hours for pain. There were no known allergies. He denied any alcohol, smoking, or illicit drug use. There was no family history of abdominal mass or malignancy. Physical examination at this time revealed a large midline, nonfluctuant, periumbilical mass that was tender to palpation. Testicular examination was normal. Interventional radiology was consulted, and together we determined that the patient would not benefit from percutaneous drainage of the mass. After a 3 -week period of watchful waiting, we decided to perform an exploratory laparotomy for potential resection of the mass. The patient consented and was booked for surgery.

The surgery occurred 3 months after the initial injury. A midline laparotomy was performed. The tumour was visible immediately upon entering the abdominal cavity. It appeared grossly to be a lymphangioma. The dissection revealed the lymphangioma to

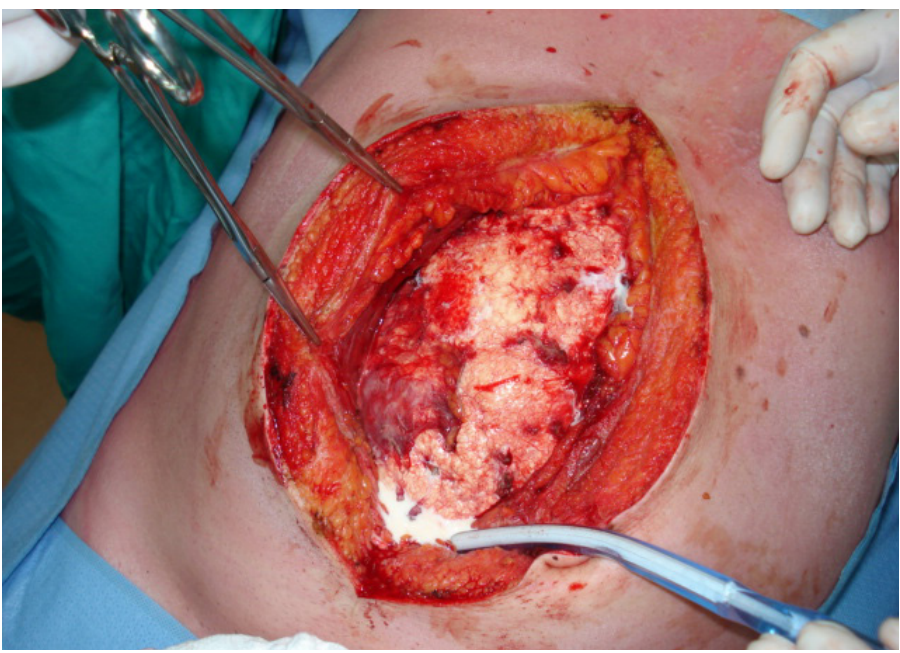

Figure 2. Intraoperative image taken after opening of the peritoneal cavity. The lymphangioma can be seen to occupy the entire field of view within the peritoneal cavity. White chyle can be seen draining from the caudal end of the lymphangioma after it was inadvertently punctured during dissection.

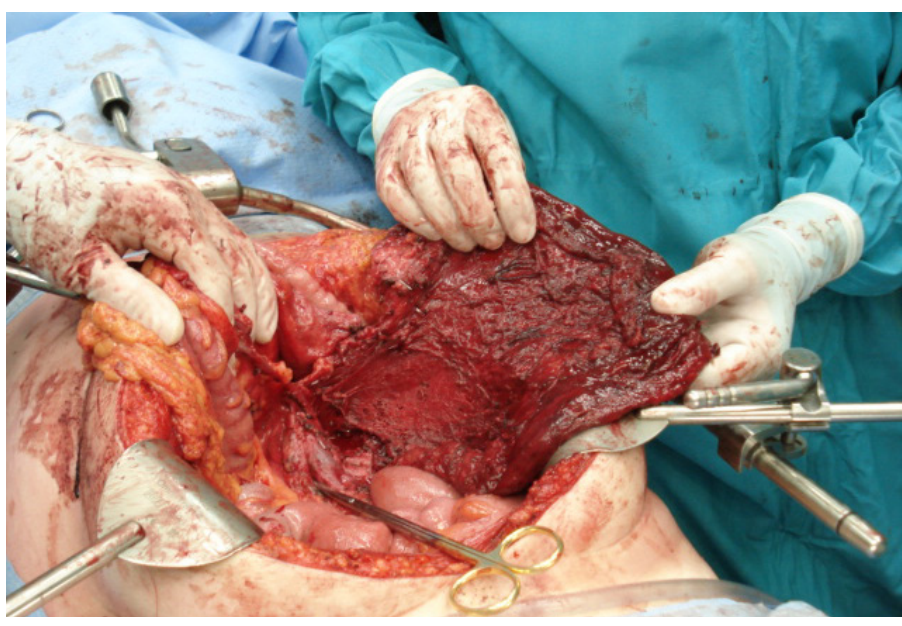

Figure 3. The lymphangioma sac can be seen as it is retracted from the peritoneal cavity. The sac can be seen to attach to the mesentery of the small bowel in addition to the mesentery of the transverse colon. originate from the small bowel mesentery at the level of the superior mesenteric artery. Additionally, there was extensive invasion of the colonic mesentery. During dissection, the tumour was inadvertently punctured; the contents were milky-white in colour and appeared chylous. We drained the cyst in vivo with simple suction. Further mobilization of the empty cyst sac revealed the stalk to be in close proximity to the superior mesenteric vein. The stalk was ligated, the cyst sac removed, and the specimen sent to the pathology department for investigation. Examination of the abdomen revealed a large defect in the colonic mesentery unsuitable for closure but with no compromise to the colonic blood supply. The abdomen was thoroughly washed and closed with no complications. Histopathology confirmed the diagnosis of lymphangioma.

The patient's postoperative course was unremarkable. He experienced complete resolution of his symptoms, and aside from postoperative pain, reported no complaints. He was discharged from the hospital on postoperative day 3 with no concerns. He continues to be symptom-free at his 2-month postoperative visit.

\section{DISCUSSION}

ETIOLOGY AND EPIDEMIOLOGY

The first case report of a mesenteric lymphangioma was by Gardner in $1852 .{ }^{4}$ Since that time, the literature on these cysts has remained sparse. The etiology of mesenteric cysts in the adult is largely unknown. The most common hypothesized cause of mesenteric lymphangioma, due to the greater prevalence of mesenteric cysts in the pediatric age group; the incidence of pediatric mesenteric lymphangioma is estimated at 1 in 20000 patients. ${ }^{3}$ Specific etiologies theorized in the pediatric population include failure of embryonic channels to join the venous system, failure of the leaves of the mesentery to fuse, and benign proliferation of ectopic lymphatics that lack communication with the normal lymphatic system. ${ }^{2}$ Presentation in the adult population is more exceptional, with estimates of the incidence of mesenteric lymphangiomas in adults between 1 in 100000 to 1 in 500000 patients. ${ }^{3}$ Theorized etiologies for development include neoplastic proliferation, lymph node degeneration, aberrant proliferation following local trauma, and benign proliferation of ectopic lymphatics. ${ }^{2}$

\section{PRESENTATION AND DIAGNOSIS}

Mesenteric cysts are often an incidental finding on imaging for other phenomena, and thus typically present asymptomatically. ${ }^{1,3,5}$ When they do present with symptoms, these often include nausea, emesis, constipation, abdominal pain, and abdominal distension; larger cysts may result in obstruction and volvulus. ${ }^{2,5}$ In a review of 162 cases, $60 \%$ of mesenteric lymphangiomas were located in the small bowel mesentery, and $24 \%$ in the large bowel mesentery. Rupture of the cyst may lead to an acute surgical abdomen requiring laparotomy. ${ }^{5}$

Diagnosis of these lesions focuses on history, physical examination, imaging, and pathology. The physical examination reveals a distended abdomen with a palpable abdominal mass. Jaundice may be seen with obstruction of biliary outflow. Ultrasound, CT, and MRI can all be used to further define and localize the mass. On imaging, mesenteric lymphangioma are often seen as solitary, 
loculated cystic lesions with sizes ranging from $4 \mathrm{~cm}$ to $30 \mathrm{~cm} .{ }^{2} \mathrm{It}$ is difficult to differentiate mesenteric lymphangiomas from duplication cysts, gastrointestinal tumours, lymphocoeles, and giant urachal cysts on imaging alone. Intraoperative examination reveals a fluid-filled sac arising from either the small bowel or large bowel mesentery, possibly with adhesions to surrounding tissues and organs. Finally, pathological examination will confirm the diagnosis of lymphangioma.

TREATMENT

Complete surgical excision of the mesenteric lymphangioma remains the mainstay of treatment. ${ }^{1,5,6}$ Incomplete resection increases the likelihood of tumour recurrence. ${ }^{1}$ Depending on the size of the cyst, laparoscopy or laparotomy may be favored; both approaches have been used with success in the literature. ${ }^{1,2,5,6}$ In $20 \%$ to $60 \%$ of cases, bowel resection with anastomosis was required to achieve complete excision of the cyst. ${ }^{5}$

\section{REFERENCES}

1. Ricketts R. Mesenteric and omental cysts. In: Grosfeld J, O’Neill J, Fonkalsrud E, Coran A, Caldamone A, eds. Pediatric Surgery. Vol 2: Mosby Inc; 2006.

2. Meitei K, Singh S, KS S. Chylomlymphatic cyst of the greater omentum presenting as abdominoscrotal swelling in a child. Indian J Urol. 2013;29:260-2.

3. Liew S, Glenn D, Storey D. Mesenteric Cyst. Aust N Z J Surg. 1994;64:741-4.

4. Gardner W. A remarkable cyst in the omentum. Transactions of the Pathological Society of London. 1852;3:1851.

5. Ghritlaharey R, More S. Chylolymphatic cyst of mesentery of terminal ileum: a case report in 8-year old boy. J Clin Diagn Res. 2014 Nov;8(11):ND05-ND07.

6. Tan J, Tan K, Chew S. Mesenteric cysts: an institution experience over 14 years and review of literature. World J Surg. 2009;33(9):161-5. 